\title{
Comprehensive Sclerotinia Stem Rot Screening of Soybean Germplasm Requires Multiple Isolates of Sclerotinia sclerotiorum
}

\author{
J. F. Willbur, S. Ding, M. E. Marks, H. Lucas, C. R. Grau, C. L. Groves, M. Kabbage, and D. L. Smith, Department of Plant Pathology,
} University of Wisconsin-Madison, Madison 53706

\begin{abstract}
Sclerotinia sclerotiorum population variability directly affects Sclerotinia stem rot (SSR) resistance breeding programs. In the north-central United States, however, soybean germplasm selection has often involved only a single isolate. Forty-four $S$. sclerotiorum isolates from Illinois, Michigan, Minnesota, Nebraska, Wisconsin, Poland, and across 11 different host species were evaluated for variation in isolate in vitro growth, in vitro oxalate production, and in planta aggressiveness on the susceptible soybean 'Williams 82 '. Significant differences $(P<0.0001)$ were detected in isolate in planta aggressiveness, in vitro growth, and in vitro oxalate production. Furthermore, diverse isolate characteristics were observed within all hosts and locations of collection. Aggressiveness was not correlated to colony growth and was only weakly correlated

$(r=0.26, P<0.0001)$ to isolate oxalate production. In addition, the host or location of collection did not explain isolate aggressiveness. Isolate oxalic acid production, however, may be partially explained by the host $(P<0.05)$ and location $(P<0.01)$ of collection. Using a representative subset of nine $S$. sclerotiorum isolates and soybean genotypes exhibiting susceptible or resistant responses (determined using a single isolate), a significant interaction $(P=0.04)$ was detected between isolates and genotypes when SSR severity was evaluated. Our findings suggest that screening of $S$. sclerotiorum-resistant soybean germplasm should be performed with multiple isolates to account for the overall diversity of $S$. sclerotiorum isolates found throughout the soybean-growing regions of the United States.
\end{abstract}

Sclerotinia sclerotiorum (Lib.) de Bary, the soilborne causal agent of Sclerotinia stem rot (SSR), is a devastating fungal pathogen affecting more than 400 host species globally (Boland and Hall 1994). Soybean (Glycine max (L.) Merrill) is one of the most economically important hosts affected by SSR (Peltier et al. 2012). In the United States, SSR ranked in the top 10 yieldreducing diseases of soybean in 2000, 2004, 2006, and 2009, and continues to significantly contribute to soybean yield reduction (Koenning and Wrather 2010; Wrather and Koenning 2009). In both 2004 and 2009, SSR accounted for an estimated 1.6 million metric ton yield reduction (Koenning and Wrather 2010; Peltier et al. 2012; Wrather and Koenning 2009). In 2011, the United Soybean Board estimated that $94 \%$ of nationwide losses occurred in the Great Lakes region. Producers lost a corresponding \$138 million according to 2011 market values (USDA-NASS 2011).

Disease control is limited by the lack of complete resistance to SSR; however, some partially resistant soybean genotypes have been identified (Boland and Hall 1987; Grau et al. 1982; Kim and Diers 2000). Traditionally, controlled-environment soybean resistance studies have been conducted with only one or two aggressive $S$. sclerotiorum isolates (Boland and Hall 1986; Hoffman et al. 2002; Kim et al. 2000; Wegulo et al. 1998). Auclair et al. (2004) proposed that such practices may be justified, and considered four different isolates of S. sclerotiorum sampled from soybean in the same geographical region as equivalent. However, the limited number of isolates used by Auclair and colleagues and the complexity of S. sclerotiorum as a pathogen, coupled with our limited understanding of the mechanisms that underpin host resistance to this pathogen, justify a broader evaluation of $S$. sclerotiorum isolates.

Furthermore, whereas overwintering sclerotia may myceliogenically germinate in the soil, the millions of ascospores generated from

Corresponding author: D. L. Smith; E-mail: damon.smith@wisc.edu

*The $\boldsymbol{e}$-Xtra logo stands for "electronic extra" and indicates that one supplementary table is published online.

Accepted for publication 2 October 2016.

C) 2017 The American Phytopathological Society the apothecial sexual fruiting body are typically the primary source of inoculum for $S$. sclerotiorum in soybean fields (Peltier et al. 2012; Willetts and Wong 1980). The limited dispersal of ascospores (Ben-Yephet and Bitton 1985) and homothallic life cycle of S. sclerotiorum (Willetts and Wong 1980) support regional clonality. Individual clones, however, may be more widely distributed by seedborne transmission of sclerotia (Hartman et al. 1998). As a result, $S$. sclerotiorum clones are considered highly dispersive, and agricultural populations may contain a conglomeration of genotypes (Anderson and Kohn 1995).

Predictably, diverse isolate populations have been documented in U.S. soybean fields (Aldrich-Wolfe et al. 2015; Koga et al. 2014; Kull et al. 2004; Petrofeza and Nasser 2012). These findings suggest that soybean genotypes should be selected for resistance using appropriately representative isolates. The importance of characterizing S. sclerotiorum isolate diversity has been investigated for dry bean resistance evaluations in Brazil (Lehner et al. 2016). Although the overall aggressiveness of 20 isolates on a susceptible or a resistant cultivar was similar, subtle differences in isolate performance between cultivars was detected. Certain isolates of S. sclerotiorum, therefore, seem better suited for evaluation of certain cultivars. S. sclerotiorum studies in Brassica spp. also considered the benefits of using a diverse set of isolates (one weakly and one strongly aggressive); however, they were unable to detect differences due to the unreliability of using only one very weak isolate (Taylor et al. 2015). As such, resistance evaluations should account for the full extent of regional variation within a pathogen population to ensure the development and release of a durable product. Therefore, pathogen diversity should be thoroughly investigated to identify appropriately representative isolates for use in resistance evaluations.

S. sclerotiorum isolate diversity has been investigated using growth characteristics, aggressiveness properties, mycelial compatibility groups (MCG), and the production of the key pathogenicity factor oxalic acid (OA) (Koga et al. 2014; Kohn et al. 1991; Kull et al. 2004). In vitro mycelial compatibility is used as an indication of isolate homogeneity and may be used to detect variation within a fungal population (Kohn et al. 1990). Multiple MCG have been detected in North America and South America (Kohn et al. 1991; Kull et al. 2004). However, MCG do not necessarily explain isolate aggressiveness at the regional level (Kull et al. 2004; Lehner et al. 2016). 
The success of resistance breeding programs is largely determined by the aggressiveness of isolates used for selection. As a result, evaluations of isolate diversity should focus on characteristics that are essential to explain S. sclerotiorum aggressiveness.

The nonhost-specific key pathogenicity factor, OA, largely contributes to the extraordinarily broad host range of S. sclerotiorum (Godoy et al. 1990; Maxwell and Lumsden 1970; Noyes and Hancock 1981). The roles of OA in S. sclerotiorum infection include $\mathrm{pH}$-dependent regulation of cell-wall-degrading enzymes (Bateman and Beer 1965), stomatal regulation (Guimarães and Stotz 2004), and suppression of host defenses (Cessna et al. 2000; Kabbage et al. 2013; Williams et al. 2011). Therefore, OA production may more accurately describe isolate aggressiveness. Indeed, OA production by $S$. sclerotiorum isolates has been previously described and was found to explain isolate aggressiveness in European red clover populations (Vleugels et al. 2013). S. sclerotiorum colony growth has also been correlated to aggressiveness in celery (Durman et al. 2003) and red clover (Vleugels et al. 2013). Therefore, colony growth and OA production are potential targets in diversity studies used to enhance SSR soybean resistance breeding programs.

The primary objective of our research was to evaluate the variability in aggressiveness of $S$. sclerotiorum isolates on soybean using a diverse collection of isolates, collected from the Midwestern United States and Poland as well as from a variety of hosts. Our secondary objective was to evaluate the use of multiple representative isolates to assess the durability of resistance responses in soybean breeding populations. Our evaluations considered a variety of explanatory factors, including colony growth characteristics and OA production, in a multipronged approach to identify essential components of isolate aggressiveness. Based on these evaluations, we investigated the use of a representative panel of $S$. sclerotiorum isolates as a tool to evaluate several soybean recombinant inbred lines (RIL) for SSR resistance. We hypothesized that isolates within our collection would exhibit variable levels of aggressiveness, in part due to pathogenicity factors such as OA but also due to specific interactions with a particular soybean genotype. Finally, exploiting the variability in S. sclerotiorum isolate characteristics will enable breeding programs to comprehensively screen soybean germplasm populations for durable SSR resistance.

\section{Materials and Methods}

Isolate collection. Between 1980 and 2013, 44 S. sclerotiorum isolates (Table 1) were collected from Indiana, Michigan, Minnesota, Nebraska, Wisconsin, and Poland. Isolates were collected from 10 hosts, including carrot (Daucus carota; $n=2$ ), celery (Apium graveolens; $n=1$ ), dwarf everlast (Helichrysum arenium; $n=3$ ), common bean (Phaseolus vulgaris; $n=2$ ), lettuce (Lactuca sativa; $n=1$ ), parsley (Petroselinum crispum; $n=1$ ), petunia (Petunia sp.; $n=1$ ), canola (Brassica napus; $n=2)$, soybean $(G$. $\max ; n=29$ ), and the common weed velvetleaf (Abutilon theophrasti; $n=2$ ). Previously, 38 of these isolates were molecularly identified as $S$. sclerotiorum in collaborative characterization studies (Baturo-Ciesniewska et al. in press). The remaining isolates were found to exhibit mycelial compatibility to verified $S$. sclerotiorum isolates (data not shown), except for isolate number 43 , which is historically well characterized as S. sclerotiorum (Amselem et al. 2011; Godoy et al. 1990; OttoHanson et al. 2011).

Isolates were grown on carrot discs to promote sclerotial formation. Sclerotia were collected using a 2.00-mm U.S. standard sieve (American Scientific), dried for $48 \mathrm{~h}$ at room temperature, and stored at $4^{\circ} \mathrm{C}$ in sterile $15-\mathrm{ml}$ polypropylene conical tubes (Becton Dickinson) until use in characterization assays. Tubes were kept in sealed plastic bags with desiccant packs (Humidity Sponge; Control Company) to promote dry storage (Pottinger et al. 2008). Stored sclerotia were surface disinfested in $10 \%$ ( $\mathrm{vol} / \mathrm{vol}$ ) bleach for $1 \mathrm{~min}$ followed by $95 \%$ ethanol for $1 \mathrm{~min}$, rinsed in sterile ultrapure Milli-Q water (EMD Millipore), blotted dry on sterile filter paper (Qualitative P8, porosity coarse; Fisher Scientific), and placed onto potato dextrose agar (PDA; Fisher Scientific) in a petri plate (100 by $15 \mathrm{~mm}$ ). Active mycelia from the leading edge of colonies were used for further analysis. Isolates were regenerated from previously dry-stored sclerotia between each repetition of the characterization assays and evaluations.

Colony growth assessment. Isolate growth was determined in vitro by monitoring PDA cultures over time. A 5-mm plug of PDA was taken from the leading edge of an actively growing colony and transferred to the center of a standard 100-by-15-mm petri plate containing PDA. Each isolate transfer was replicated three times and each replicate was randomized in stacks placed in an incubator with a 14-h photoperiod at $16^{\circ} \mathrm{C}$ for the duration of the assay. An OAdeficient (A2) mutant was included to determine the role of OA in in vitro growth (Godoy et al. 1990). Mycelial growth was measured along two axes ( $\mathrm{x}$ and $\mathrm{y}$, at a right angle extending from the center of the plate) once a day for three to four consecutive days until mycelia reached the edge of the agar plate. Standardized areas under the growth curves (STAUGC) were used for statistical analyses. The STAUGC is analogous to the standardized area under the disease progress curve (STAUDPC) as described by Campbell and Madden (1990). The colony growth assessment was repeated once after regeneration of isolates from dry-stored sclerotia.

OA production. OA production was determined in vitro in potato dextrose broth (PDB; EMD Chemicals Inc.) cultures. A 5-mmdiameter plug was transferred from the leading edge of an actively growing culture of each isolate to $25 \mathrm{ml}$ of sterile PDB in $125-\mathrm{ml}$ Erlenmeyer flasks. Broth cultures were incubated at room temperature $\left(21\right.$ to $\left.24^{\circ} \mathrm{C}\right)$ in complete darkness for 3 days. Cultures were agitated by hand twice daily to ensure that mycelia remained suspended in the broth. After 3 days, 5-ml samples of culture broth were collected for OA determination. Remaining cultures were vacuum filtered and mycelia were collected, weighed, dried at $37^{\circ} \mathrm{C}$ for 2 days, and reweighed. Dry mycelial weights were used to adjust OA concentrations for each isolate to a per-milligram unit for statistical analyses.

OA levels were quantified using an enzymatic assay kit (Trinity Biotech plc, Bray, Co.). Broth samples were diluted 1:1 using kit diluent and adjusted to a $\mathrm{pH}$ of 6 to 7 with either $1 \mathrm{~N}$ hydrochloric acid or $1 \mathrm{~N}$ sodium hydroxide. Enzymatic assays were conducted on activated charcoal purified samples according to the instructions in the kit. Resulting sample reactions were transferred to a 96-well microplate and absorbencies at $590 \mathrm{~nm}$ were recorded using a Bio-Rad iMark (Bio-Rad Laboratories) microplate reader. Two negative controls - broth cultures with a sterile PDA plug only (technical control) or a broth culture with an OA-deficient mutant-were included in all experiments; absorbencies were corrected with the mutant background. An oxalate standard curve produced from $0.00,0.25$, $0.50,1.00,2.00$, and $4.00 \mathrm{mmol} / \mathrm{liter}$ standards was used to convert sample absorbencies to oxalate concentrations. Resulting concentrations were adjusted to a milligram unit using the corresponding mycelial dry weights. All isolates were replicated three times and the entire experiment was repeated once. Isolates were regenerated from dry-stored sclerotia between each repetition.

Aggressiveness evaluations. Susceptible Williams 82 soybean was challenged with all $S$. sclerotiorum isolates to evaluate isolate aggressiveness in planta. Soybean seed were planted in $15.25-\mathrm{cm}$ pots, approximately $4 \mathrm{~cm}$ deep in moist potting mix (Sun Gro Horticulture), and grown in a growth room with a 14-h photoperiod, set to $22^{\circ} \mathrm{C}$ during the day and $18^{\circ} \mathrm{C}$ at night. Seed were watered daily and fertilized once a week (Miracle-Gro; Scotts Miracle-Gro Products). Soybean plants grown to the V4 growth stage (four fully emerged trifoliates) were used to conduct aggressiveness evaluations.

Isolate inoculum was prepared by transferring a 5-mm plug of agar from the leading edge of an actively growing PDA culture on a standard petri plate to a deep petri plate $(100$ by $25 \mathrm{~mm}$; Lab-Tek Nunc, Sigma-Aldrich) with PDA. Inoculations were conducted using a previously described cut-petiole technique (Peltier and Grau 2008). Using 1,000- $\mu$ l pipet tips (Fisher Scientific), a 1.5-cm-thick agar core was collected from the leading edge of mycelia on each inoculum plate. Second trifoliate leaflets were excised such that petioles were 2.5 to $3 \mathrm{~cm}$ in length. Petioles were inserted into pipet tips of inoculum and positioned so that mycelia and cut-petiole tissue were in direct contact. 
Isolate inoculations were performed in triplicate (three replicates) and soybean plants were arranged in a randomized complete block design (RCBD). An OA-deficient mutant was also included in every experiment to determine the role of OA in planta. Stem lesions were measured daily using a digital caliper (Thermo Fisher Scientific) for a duration of 7 to 14 days postinoculation (depending on repetition). Square roots of STAUDPC were used for statistical analyses of isolate aggressiveness. The square root transformation was used to normalize the data; however, back-transformed means are presented graphically. Each aggressiveness evaluation was repeated once, and isolates were regenerated from previously dry-stored sclerotia between each repetition.

STAUDPC results from these aggressiveness assays were used to define "aggressive" and "mildly aggressive" isolate designations. Isolates with an STAUDPC value not statistically different from the isolate with the highest STAUDPC and statistically different from the isolate with the lowest STAUDPC were considered aggressive. Isolates with an STAUDPC value greater than 0.00 but not statistically different from the isolate with the lowest STAUDPC were considered mildly aggressive.

Multi-isolate-soybean germplasm evaluations. To investigate the response of soybean germplasm to a variety of S. sclerotiorum isolates, a preliminary screen was conducted in a greenhouse at the West Madison Agricultural Research Station, Wisconsin. Two putatively resistant experimental lines (determined from preliminary testing; data not shown), 91-38 (classified as moderately resistant; MR) and 91-145 (classified as highly resistant; HR); one susceptible (S) experimental line, 91-44; and an S check, Williams 82, were selected for multi-isolate challenge. Line phenotypes were determined using the previously described cut-petiole method with a single isolate, number 30 . These lines were evaluated using a modified 0 -to-4 rating scale, where $0=$ no lesion on the main stem, $1=$ small lesion (approximately $<1 \mathrm{~cm})$ on the main stem, $2=$ considerable lesion $(\geq 1 \mathrm{~cm})$ development on the main stem but no associated foliar wilt, $3=$ considerable lesion development and irreversible foliar wilt, and $4=$ lesion development resulting in poor pod fill and plant death (Grau et al. 1982). All germplasm lines (91-38, 91-145, and 91-44) were generated from the initial cross of the soybean cyst nematoderesistant line LN89-5717 (Nickell et al. 1994) and the partially SSR-resistant line W04-1002 (Peltier and Grau 2008). Experimental lines were of the F8:12 generation (i.e., from the fourth subsequent progeny of a single F8-derived soybean plant). Lines were considered highly inbred with limited genetic diversity. Soybean seed were

Table 1. Collection information of 44 Sclerotinia sclerotiorum isolates ${ }^{\mathrm{z}}$

\begin{tabular}{|c|c|c|c|c|c|}
\hline Isolate number & Location & Host & Common name & Year & Collector, citation \\
\hline 1 & Wisconsin & Glycine $\max$ & Soybean & 2012 & D. Smith \\
\hline 2 & Wisconsin & G. $\max$ & Soybean & 2012 & D. Smith \\
\hline 3 & Wisconsin & G. $\max$ & Soybean & 2012 & D. Smith \\
\hline 5 & Illinois & G. $\max$ & Soybean & - & C. Bradley \\
\hline 6 & Illinois & Abutilon theophrasti & Velvetleaf & - & C. Bradley \\
\hline 7 & Illinois & A. theophrasti & Velvetleaf & - & C. Bradley \\
\hline 8 & Michigan & G. $\max$ & Soybean & - & M. Chilvers \\
\hline 9 & Michigan & G. $\max$ & Soybean & - & M. Chilvers \\
\hline 10 & Michigan & G. $\max$ & Soybean & - & M. Chilvers \\
\hline 15 & Wisconsin & G. $\max$ & Soybean & - & C. Grau \\
\hline 16 & Wisconsin & G. $\max$ & Soybean & - & C. Grau \\
\hline 17 & Wisconsin & G. $\max$ & Soybean & - & C. Grau \\
\hline 18 & Wisconsin & G. $\max$ & Soybean & - & C. Grau \\
\hline 19 & - & G. $\max$ & Soybean & 2012 & C. Fritz \\
\hline 20 & - & G. $\max$ & Soybean & 2012 & C. Fritz \\
\hline 21 & - & G. $\max$ & Soybean & 2012 & C. Fritz \\
\hline 22 & Wisconsin & G. $\max$ & Soybean & - & C. Grau \\
\hline 23 & Wisconsin & G. $\max$ & Soybean & 2003 & C. Grau \\
\hline 24 & Wisconsin & G. $\max$ & Soybean & 2012 & C. Grau/K. Lackermann \\
\hline 25 & Wisconsin & G. $\max$ & Soybean & 2012 & C. Grau/K. Lackermann \\
\hline 26 & Wisconsin & G. $\max$ & Soybean & 2000 & C. Grau \\
\hline 27 & Wisconsin & G. $\max$ & Soybean & - & C. Grau \\
\hline 28 & Wisconsin & G. $\max$ & Soybean & - & C. Grau \\
\hline 29 & - & G. $\max$ & Soybean & 2012 & C. Fritz \\
\hline 30 & Wisconsin & G. $\max$ & Soybean & 2012 & C. Grau/K. Lackermann \\
\hline 31 & Wisconsin & G. $\max$ & Soybean & - & C. Grau \\
\hline 32 & Wisconsin & G. $\max$ & Soybean & 2012 & C. Grau/K. Lackermann \\
\hline 33 & Wisconsin & G. $\max$ & Soybean & - & C. Grau \\
\hline 34 & - & G. $\max$ & Soybean & 2012 & C. Fritz \\
\hline 43 & $\mathrm{NE}$ & Phaseolus vulgaris & Common bean & 1980 & Godoy et al. 1990 \\
\hline 44 & Wisconsin & P. vulgaris & Common bean & 2009 & D. Malvick \\
\hline 45 & Minnesota & Petunia sp. & Petunia & 2009 & D. Malvick \\
\hline 46 & Minnesota & G. $\max$ & Soybean & 2013 & D. Malvick \\
\hline 47 & Minnesota & G. $\max$ & Soybean & 2013 & D. Malvick \\
\hline 57 & Poland & Brassica napus & Canola & 2011 & A. Baturo-Ciesniewska \\
\hline 58 & Poland & Helichrysum arenarium & Dwarf everlast & 2012 & A. Baturo-Ciesniewska \\
\hline 59 & Poland & H. arenarium & Dwarf everlast & 2012 & A. Baturo-Ciesniewska \\
\hline 60 & Poland & H. arenarium & Dwarf everlast & 2012 & A. Baturo-Ciesniewska \\
\hline 61 & Poland & Daucus carota & Carrot & 2012 & A. Baturo-Ciesniewska \\
\hline 62 & Poland & Brassica napus & Canola & 2013 & A. Baturo-Ciesniewska \\
\hline 63 & Poland & D. carota & Carrot & 2013 & A. Baturo-Ciesniewska \\
\hline 64 & Poland & Apium graveolens & Celery & 2012 & A. Baturo-Ciesniewska \\
\hline 65 & Poland & Petroselinum crispum & Parsley & 2012 & A. Baturo-Ciesniewska \\
\hline 66 & Poland & Lactuca sativa & Lettuce & 2013 & A. Baturo-Ciesniewska \\
\hline
\end{tabular}

${ }^{\mathrm{z}}$ Symbol: - indicates information unknown; highly likely that all isolates with unknown location information were collected in Wisconsin. 
planted in $15.25-\mathrm{cm}$ peat pots approximately $4 \mathrm{~cm}$ deep in moist potting mix (Sun Gro Horticulture), watered daily, and fertilized twice a week (Scotts Peters Professional Peat-Lite Special 20-10-20; ScottsSierra Horticultural Products Co.) until the V4 growth stage.

Representative isolates were selected from the previously characterized 44 isolates. Four strongly aggressive isolates, four mildly aggressive isolates, and the original single isolate used in previous screens (a total of nine isolates) were included for resistance evaluations (Table 2). Cut-petiole inoculations were conducted as described previously. Inoculated plants were arranged in a RCBD to accommodate all four-by-nine factorial treatment combinations. Isolate-line interactions were evaluated in triplicate (three replicates) and repeated once. Isolates were regenerated from sclerotia between each repetition. Stem lesions were measured every 2 or 3 days for 13 days postinoculation using a digital caliper, as described above.

An additional repetition (for a total of three repetitions) was conducted in the same greenhouse at the West Madison Agricultural Research Station, Wisconsin using only the 91-145, 91-38, and 91-44 RIL. Three soybean seeds were planted equidistant from each other in $15.25-\mathrm{cm}$ peat pots approximately $4 \mathrm{~cm}$ deep in moist potting mix (Sun Gro Horticulture). The plants were watered daily and fertilized as previously described until the V5 growth stage. Second-trifoliate petioles of all plants in each pot were excised to a length of $2.5 \mathrm{~cm}$ and inoculated using the pipet tip method described previously. All nine isolates of $S$. sclerotiorum used in the preceding screens were regenerated from dry-stored sclerotia and included in the evaluation. Stem lesions were measured 5,10 , and 15 days postinoculation using the previously described digital calipers. STAUDPC was analyzed to evaluate germplasm resistance reactions to a variety of isolates in all greenhouse screens. A square-root transformation was used to normalize STAUDPC data; however, back-transformed means were presented graphically.

Statistical analyses. Correlations between isolate colony growth, OA production, and aggressiveness were determined using Pearson correlation coefficients generated in SAS (v. 9.3; SAS Institute) using the PROC CORR procedure. Correlations were evaluated at the $\alpha=0.05$ significance level.

Isolate variation by in vitro growth (STAUGC), OA production, and aggressiveness (STAUDPC) were used as dependent variables in generalized linear mixed model (GLIMMIX) analysis of variance using SAS (v. 9.3; SAS Institute). Host and location of collection were also used to explain isolate variation. Replicate and repetition (run) were combined in a nested random effect [rep(run)].

Raw data from the previous analysis were extracted for the nine isolates selected for the multi-isolate evaluations and analyzed as a subset using the SAS (v. 9.3; SAS Institute) GLIMMIX procedure. Isolate aggressiveness (STAUDPC) was the only dependent variable used in this subset analysis. Repetition (run) and replicate were treated as a nested random effect [rep(run)].

A similar GLIMMIX analysis was performed for the multi-isolategermplasm line experiment. Isolate and germplasm line were treated as fixed effects and repetition (run) and replicate were combined in a nested random effect [rep(run)]. In order to maintain a balanced design, all Williams 82 observations were removed from the pooled analyses of runs 1, 2, and 3. Differences between and within the remaining 91-145, 91-38, and 91-44 RIL were determined at the $\alpha=0.05$ significance level.

The extracted Williams 82 observations from runs 1 and 2 of the multi-isolate-soybean germplasm evaluations were used to validate characterization of the selected isolates in the preceding growthroom evaluations. Comparison of growth-room and greenhouse results of isolate aggressiveness on Williams 82 soybean was conducted using STAUDPC values generated in a GLIMMIX analysis in SAS (v. 9.3; SAS Institute), as previously described. Isolate aggressiveness groupings were evaluated using Fisher's least significant difference (LSD) letter groupings; means with the same letter were not considered different at the $\alpha=0.05$ significance level.

\section{Results}

Diversity of $\boldsymbol{S}$. sclerotiorum isolates. Isolate STAUGC, oxalate production, and aggressiveness, measured as STAUDPC, were used to characterize the diversity of this $S$. sclerotiorum isolate collection. In vitro growth, in vitro OA production, and in planta aggressiveness were significantly different $(P<0.0001)$ among all isolates (Fig. 1).

All wild-type isolates exhibited characteristic growth on PDA; the mean STAUGC value of this collection $(n=45)$ was 14.35 , with a minimum of 7.23 and a maximum of 18.07 (Fig. 1A). All isolates, except the OA-deficient mutant, secreted acid levels detected by a $\mathrm{pH}$ change from 7.10 (mean of technical control) to between 6.01 and 6.97 over the 3-day incubation period (data not shown). The mean OA concentration produced by these isolates $(n=45)$ was $0.13 \mathrm{mmol} / \mathrm{liter} / \mathrm{mg}$, with a minimum of $6.34 \times 10^{-14} \mathrm{mmol} / \mathrm{liter} / \mathrm{mg}$ and a maximum of $0.25 \mathrm{mmol} / \mathrm{liter} / \mathrm{mg}$ (Fig. 1B). In aggressiveness assays, Williams 82 stems inoculated with wild-type isolates exhibited characteristic lesions ranging from light tan to dark brown. The mean STAUDPC value of the isolates evaluated $(n=45)$ was 16.74 , with a minimum value of $<0.01$ and a maximum of 31.39 (Fig. 1C). Minimum values of all three assays resulted from the OA-deficient mutant, denoted as isolate $\mathrm{A}$.

Correlations with aggressiveness. The OA-deficient mutant, denoted as isolate $\mathrm{A}$, exhibited a delayed growth phenotype in vitro (Fig. 1A). However, as expected, OA mutant in vitro oxalate concentrations were not significantly different from background absorbance levels and are presented as $0.00 \mathrm{mmol} / \mathrm{liter} / \mathrm{mg}$ (Fig. 1B). On the host plant, the OA mutant was unable to progress the length of the petiole and no lesion formed on the stem, resulting in an STAUDPC of 0.00 (Fig. 1C). These results suggest a potential correlation between in vitro oxalate production and in planta pathogenicity but do not support a correlation between in vitro growth and aggressiveness.

A weak positive correlation $(r=0.26, P<0.0001)$ was identified between OA production and aggressiveness (Fig. 2). STAUGC was very weakly correlated $(r=0.13, P<0.05)$ with aggressiveness in these experiments. Based on these results, in vitro isolate growth and $\mathrm{OA}$ production do not accurately describe isolate aggressiveness. Thus, despite the importance of OA in S. sclerotiorum pathogenicity, as shown with the OA-deficient mutant, natural variations in OA levels among field isolates did not fully explain the variability in isolate aggressiveness.

Effect of host or location of collection. Host and location of collection were used to further describe variation of isolate in vitro $\mathrm{OA}$ production and in planta aggressiveness. The diversity of isolate OA production in our collection was explained by plant host $(P<0.05)$ (Fig. 3) and location of collection $(P<0.01)$ (Fig. 4). Isolates

Table 2. Collection information of nine Sclerotinia sclerotiorum isolates selected for multi-isolate resistance evaluations of soybean germplasm ${ }^{\mathrm{y}}$

\begin{tabular}{lcll}
\hline Isolate number & STAUDPC $^{\mathbf{z}}$ & \multicolumn{1}{c}{ Host } & Location \\
\hline 47 & $2.39 \mathrm{~d}$ & Soybean & Minnesota \\
60 & $6.21 \mathrm{~cd}$ & Dwarf everlast & Poland \\
15 & $6.72 \mathrm{~cd}$ & Soybean & Wisconsin \\
3 & $9.22 \mathrm{~cd}$ & Soybean & Wisconsin \\
62 & $25.64 \mathrm{ab}$ & Canola & Poland \\
19 & $25.13 \mathrm{ab}$ & Soybean & - \\
10 & $29.43 \mathrm{ab}$ & Soybean & Michigan \\
20 & $31.38 \mathrm{a}$ & Soybean & - \\
30 & $14.61 \mathrm{bc}$ & Soybean & Wisconsin \\
LSD & 1.69 & - & - \\
\hline
\end{tabular}

y Isolates were selected based on consistent standardized areas under the disease progress curves (STAUDPC) across two growth-room evaluations. Means are presented for four mildly aggressive isolates (numbers 47, 60, 15, and 3), four strongly aggressive isolates (numbers 62, 19, 10, and 20), and the original isolate used in Wisconsin soybean resistance evaluations (number 30). Means followed by the same letter are not significantly different based on Fisher's least significant difference (LSD; $\alpha=0.05$ ); - indicates information unknown; highly likely that all isolates with unknown location information were collected in Wisconsin.

${ }^{\mathrm{z}}$ Aggressiveness data were extracted from the existing standardized area under the disease progress curves (STAUDPC) data set, from experiments conducted on Williams 82 soybean, and analyzed as a subset. 
collected from canola $(n=2)$ and parsley $(n=1)$ produced the highest and lowest OA levels, respectively. Additionally, isolates from Poland $(n=10)$ and Michigan $(n=3)$ displayed the highest OA concentrations, whereas isolates collected from Minnesota $(n=3)$ produced the lowest concentrations. Variability in isolate aggressiveness was observed across all hosts (Fig. 5) and locations (Fig. 6); aggressiveness on a susceptible soybean cultivar, however, was not explained by either host or location.

Representative subset of isolates. Representative isolates were selected based on in planta aggressiveness on a susceptible soybean
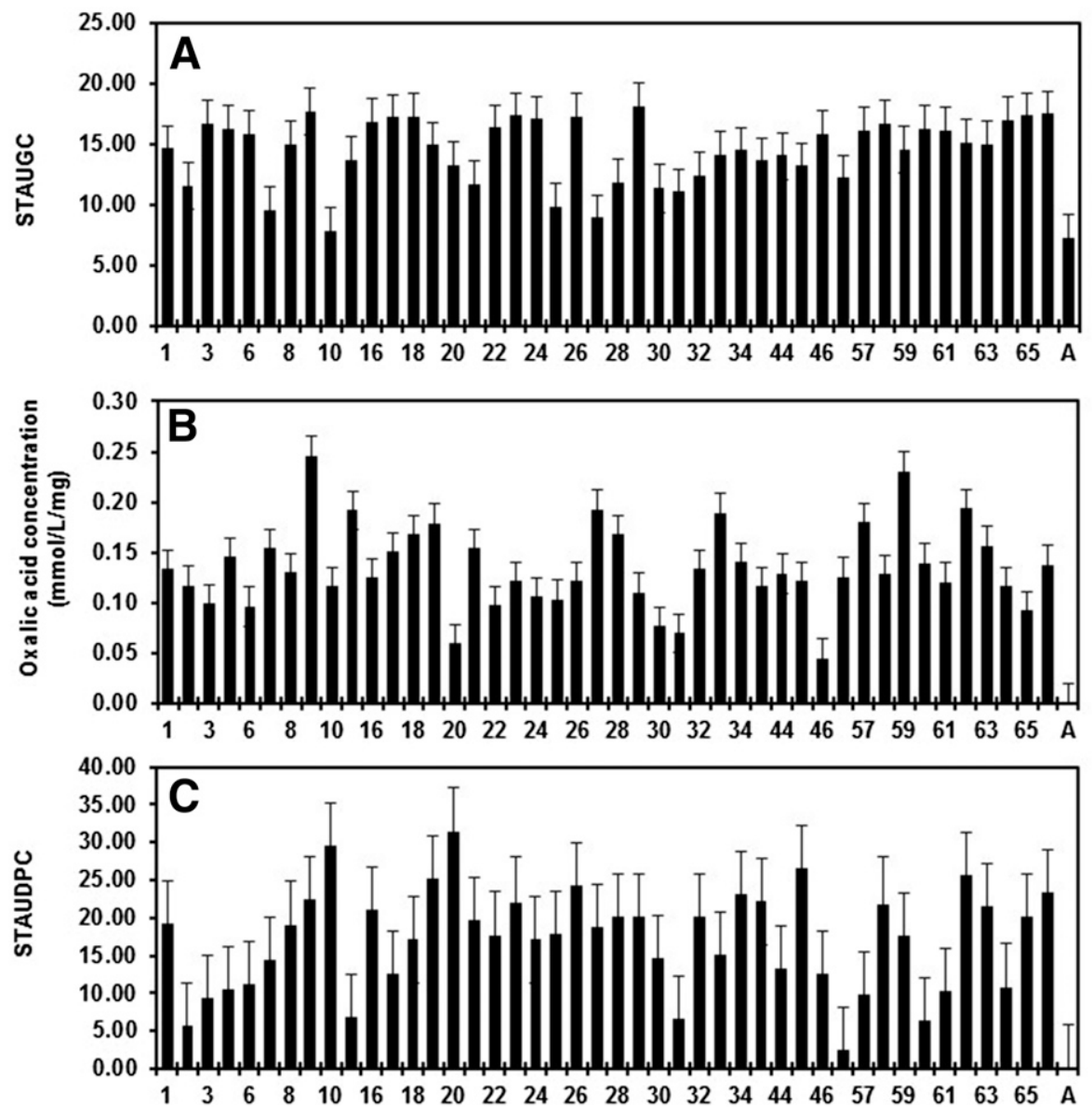

Fig. 1. Diversity in Sclerotinia sclerotiorum isolate A, in vitro growth on potato dextrose agar, measured as standardized areas under the growth curves (STAUGC); B, in vitro oxalic acid production in potato dextrose broth, measured oxalate concentrations determined by spectrophotometric absorbencies at $590 \mathrm{~nm}$; and $\mathbf{C}$, aggressiveness on susceptible Williams 82 soybean, measured as standardized areas under the disease progress curves (STAUDPC). An oxalic-acid-deficient mutant (bar A) was included in every experiment. Isolate differences in STAUGC, oxalic acid production, and STAUDPC are significant $(P<0.0001)$. Error bars represent standard errors of the mean values, based on three replicates from two repetitions, for each isolate.

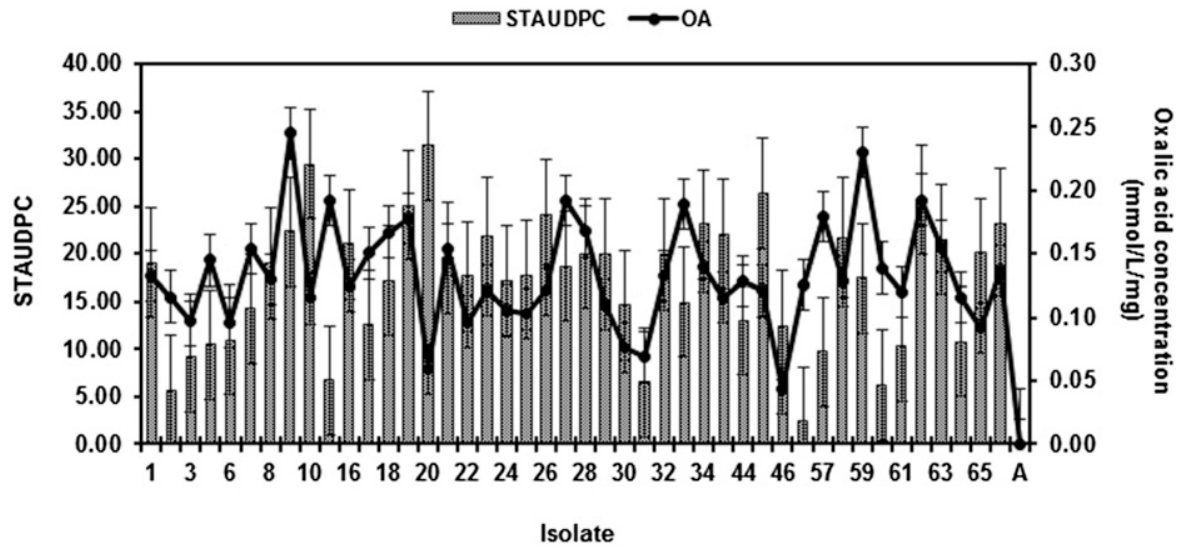

Fig. 2. Correlations between Sclerotinia sclerotiorum isolate aggressiveness on susceptible Williams 82 soybean, measured as standardized area under the disease progress curves (STAUDPC), and in vitro oxalic acid (OA) production (shown on the secondary axis), determined using spectrophotometric absorbencies at $590 \mathrm{~nm}$ and an oxalate standard curve. An OA-deficient mutant (bar A) was included in every experiment. A weakly positive correlation was detected between mean STAUDPC and OA concentrations $(r=0.26, P<0.0001)$. Error bars represent standard errors of the mean values from three replicates for each isolate. 
cultivar, as measured in previous experiments (STAUDPC). Isolate colony growth, OA production, host of origin, and location of collection were not considered due to the lack of any significant effects on isolate aggressiveness. Therefore, four strongly (numbers 10, 19, 20, and 62) and four mildly (numbers 3, 15, 47, and 60) aggressive isolates were selected to inoculate soybean RIL (Table 2). STAUDPC replicates from both repetitions $(n=6)$ were extracted and pooled for each isolate to determine mean differences at the $\alpha=0.05$ level. Based on Fisher's LSD $(\alpha=0.05)$, selected isolates within an aggressiveness grouping (i.e., strong or mild) were not significantly different from one another; however, isolates from different groups were significantly different on susceptible Williams 82 soybean.

Comparison of growth-room and greenhouse evaluations. Comparisons were conducted to validate isolate aggressiveness designations determined in growth-chamber experiments. According to LSD letter groupings, the majority of the isolates clustered similarly to previous growth-room evaluations (Supplementary Table S1). Seven of the nine isolates successfully exhibited responses that corresponded to the previously determined aggressiveness designations. The letter groupings indicated that three of the four mildest isolates (numbers 47, 60, and 15) were not significantly different from one another and had the lowest STAUDPC means. Likewise, four of the strongly aggressive isolates (numbers 20,19, 10, and 62) were not significantly different from one another and had among the highest STAUDPC means. Only isolates 3 and 30 appeared considerably more aggressive in greenhouse evaluations.
Multi-isolate-soybean germplasm evaluations. Response of several soybean RIL was previously tested using a single isolate of S. sclerotiorum (number 30; data not shown). In this study, resistant soybean lines 91-145 (HR) and 91-38 (MR), susceptible line 91-44 $(\mathrm{S})$, and Williams $82(\mathrm{~S})$ were reevaluated using the original isolate used for selection (number 30) and four strongly (numbers 10, 19, 20 , and 62), and four mildly (numbers $3,15,47$, and 60) aggressive isolates (Table 2). The isolate-line interaction was significant $(P=0.04)$ in the pooled STAUDPC analyses.

Based on the mean STAUDPC of 7.19, line 91-145 (HR) did appear highly resistant. Line 91-38 (MR) and 91-44 (S), however, do not exhibit significantly different reactions from each other based on STAUDPC means of 11.88 and 15.02, respectively. Threshold STAUDPC values were determined from these means to describe the individual isolate-line reactions independent of previous line designations (HR, MR, or S). Germplasm reactions with STAUDPC $\leq$ 10 were considered resistant, STAUDPC $>10$ but $\leq 15$ were considered moderately susceptible, and STAUDPC $>15$ were considered susceptible.

Overall, line 91-145 (HR) successfully exhibited resistant reactions to seven of the nine isolates (Fig. 7A). However, isolates 10 and 20 caused a moderately susceptible (MS) and S reaction, respectively, in line 91-145. Line 91-38 (MR) only appeared noticeably resistant against four of the nine isolates (Fig. 7B). Use of isolates 3, 10, 15,19 , and 62 against soybean line 91-38 elicited MS to $\mathrm{S}$ reactions. Additionally, line 91-44 (S) only checked as S using seven of the nine

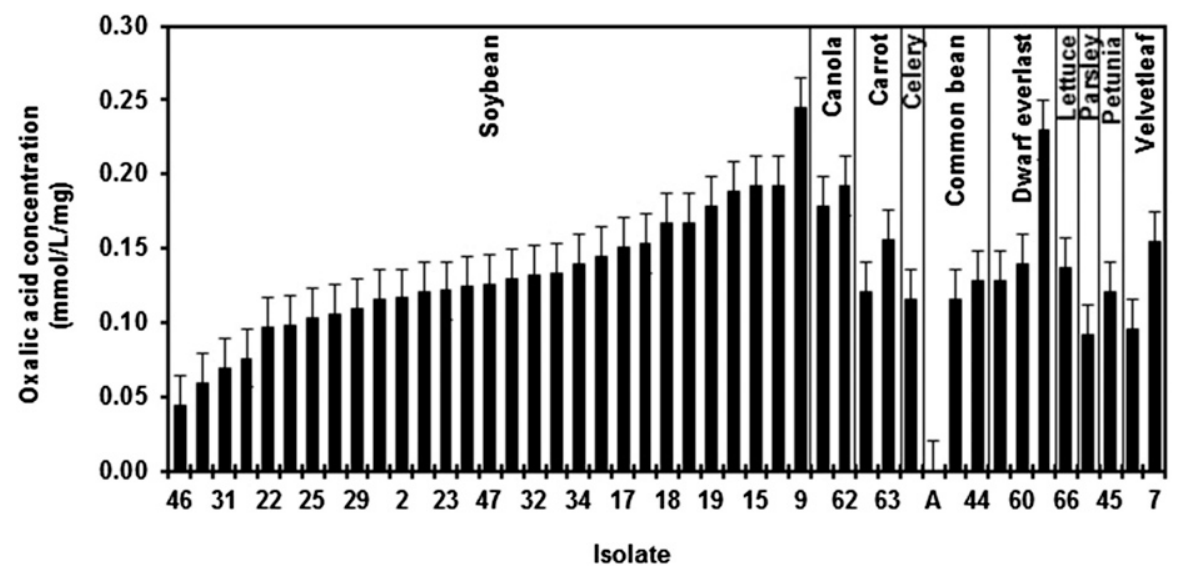

Fig. 3. Significant effect $(P<0.05)$ of plant host of origin on isolate in vitro oxalic acid production, determined using spectrophotometric absorbencies at $590 \mathrm{~nm}$ and an oxalate standard curve. Solid bars represent means of three replicates from two repetitions. Error bars represent standard errors of the mean values for each isolate.

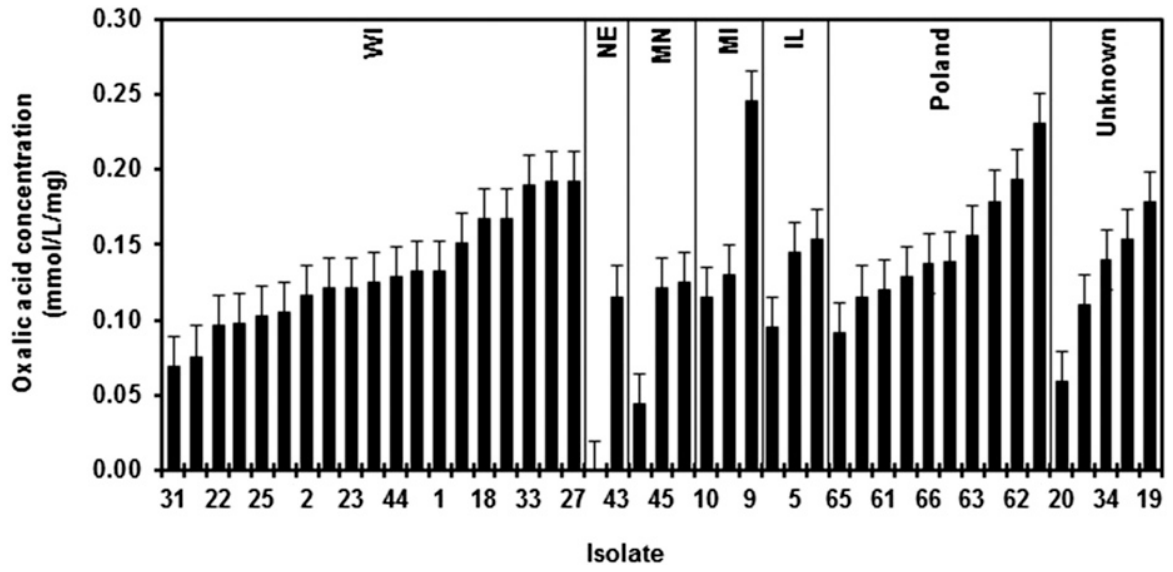

Fig. 4. Significant effect $(P<0.01)$ of location of collection on Sclerotinia sclerotiorum isolate in vitro oxalic acid production as determined using spectrophotometric absorbencies at $590 \mathrm{~nm}$ and an oxalate standard curve. $\mathrm{WI}=$ Wisconsin, $\mathrm{NE}=$ Nebraska, $\mathrm{MN}=$ Minnesota, $\mathrm{MI}=$ Michigan, and IL = Illinois. Solid bars represent means of three replicates two repetitions. Error bars represent standard errors of the mean values for each isolate. 
isolates (Fig. 7C). The use of isolates 47 and 60 resulted in a resistant response. Therefore, these data show that, although the overall response of the soybean lines tested in this study was predictable and confirmed previous trends, SSR severity in some instances was predicated by specific line-isolate interactions. Some isolates are better suited to produce higher SSR severity on specific soybean lines regardless of perceived aggressiveness (Fig. 7), thus justifying the use of multiple $S$. sclerotiorum isolates when evaluating SSR levels in breeding lines.

\section{Discussion}

The overall results of our study suggest that the representative S. sclerotiorum isolate collection presented here contained a range of mildly to strongly aggressive isolates on soybean. These findings corroborate other reports of variation in isolate aggressiveness (Kull et al. 2004; Li et al. 2008; Vleugels et al. 2013). As previously described, differences were also observed in $S$. sclerotiorum isolate OA production (Durman et al. 2005; Li et al. 2008; Vleugels et al. 2013). Isolate variability in OA levels, however, did not completely explain the variability in in planta aggressiveness. A strong correlation between in vivo oxalate production and aggressiveness was reported in red clover $(r=0.46, P=0.010)$ (Vleugels et al. 2013) and sunflower $(r=0.74, P<0.01)$ (Li et al. 2008). However, the weak ( $r=0.26, P<0.0001)$ correlation we observed is more consistent with previous conclusions that isolate aggressiveness in common bean stem inoculations was not explained by the oxaloacetate acetylhydrolase $(\mathrm{OAH})$ haplotype which is involved in OA biosynthesis (Lehner et al. 2016). Thus, it is conceivable that, although OA is an important virulence factor, its production beyond a specific threshold does not always correlate with increased aggressiveness. In addition to OA, S. sclerotiorum may also require additional pathogenicity factors for successful infection, including cell-wall-degrading enzymes, toxins, and proteinaceous effectors (Guyon et al. 2014; Kabbage et al. 2015). A recent study showed that $\mathrm{OAH}$ mutants were able to induce restricted lesions on detached leaves despite their inability to produce OA (Liang et al. 2015). Clearly, OA is not the only contributing factor to $S$. sclerotiorum aggressiveness. It is not surprising, therefore, that a single pathogenicity factor, while important, does not solely describe isolate aggressiveness. Alternatively, OA production by S. sclerotiorum may be conditioned by environmental factors within the infection court. Thus, future studies should consider OA levels in the context of the host plant, in addition to in vitro production.

Our S. sclerotiorum isolate collection exhibited significant variation in isolate radial colony growth in accordance with previous

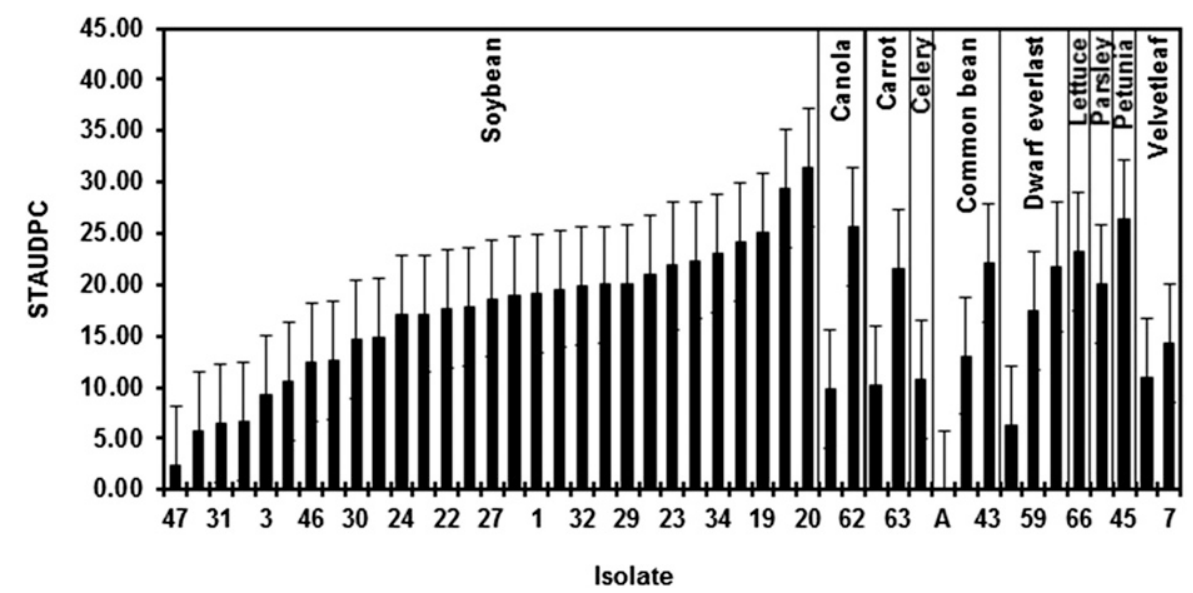

Fig. 5. Effect of host origin on Sclerotinia sclerotiorum isolate in planta aggressiveness on susceptible Williams 82 soybean, measured as standardized area under the disease progress curves (STAUDPC). Growth-room evaluations were conducted by collecting daily stem lesion measurements (in millimeters) for 7 to 14 days postinoculation. Solid bars represent means of three replicates from two repetitions. The effect of host origin within each run was not significant $(P>0.05)$. Error bars represent standard errors of the mean values for each isolate.

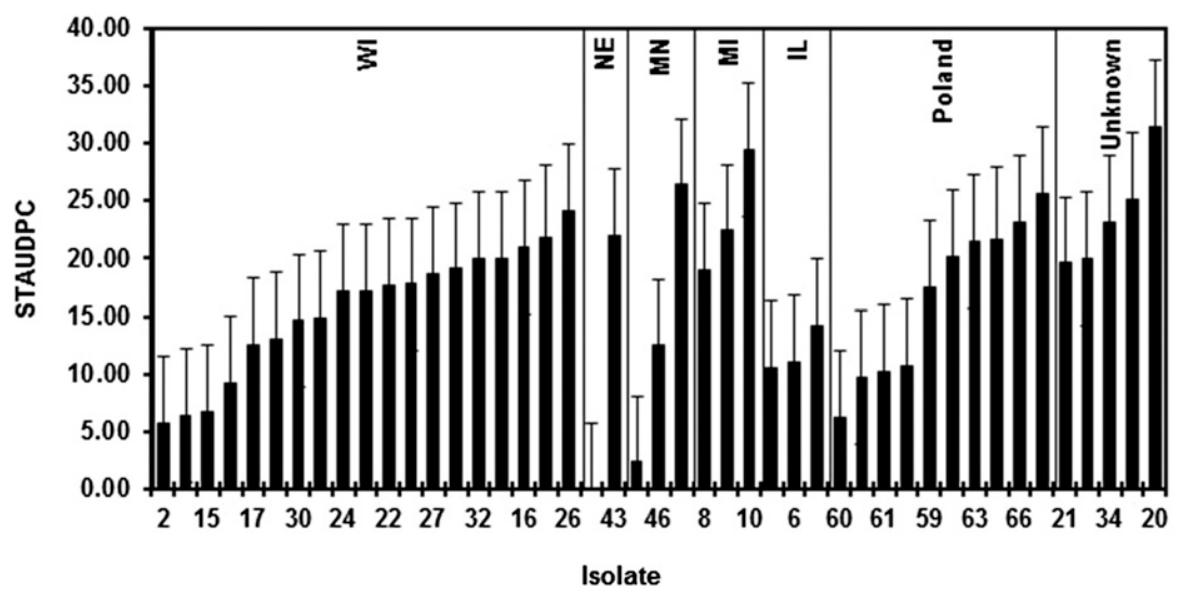

Fig. 6. Effect of location of collection on Sclerotinia sclerotiorum isolate in vivo aggressiveness on susceptible Williams 82 soybean, measured as standardized areas under the disease progress curves (STAUDPC). Growth-room evaluations were conducted by collecting daily stem lesion measurements (in millimeters) for 7 to 14 days postinoculation. WI = Wisconsin, $\mathrm{NE}=$ Nebraska, $\mathrm{MN}=$ Minnesota, $\mathrm{MI}=$ Michigan, and IL = Illinois. Solid bars represent means of three replicates from two repetitions. The effect of location within each run was not significant $(P>0.05)$. Error bars represent standard errors of the mean values for each isolate. 
reports (Garg et al. 2009; Li et al. 2008; Vleugels et al. 2013); however, only a weak correlation was observed between colony growth and aggressiveness in our study. Previous studies reported the presence of aggressiveness-correlated colony growth in detached celery petiole assays (Durman et al. 2003) and in red clover leaf and plant assays (Vleugels et al. 2013). Other studies, however, reported no significant correlation between colony radial growth and aggressiveness on either Brassica sp. cotyledons (Garg et al. 2009) or detached sunflower leaves (Li et al. 2008). Our results provide additional evidence that the complexity of $S$. sclerotiorum isolate aggressiveness is poorly correlated with in vitro growth characteristics. This is not surprising, considering that disease outcome is largely based on virulence factors and host responses during pathogenic development compared with saprophytic growth.

The host and location of collection did not explain the variation observed in isolate aggressiveness. The overall variation in isolate aggressiveness was well represented within most hosts and locations. Differences between hosts or locations, however, were difficult to distinguish due to the unbalanced distribution of isolates within our collection (i.e., approximately half of the isolates originated from Wisconsin soybean plants). Despite this limitation, our findings support other reports of variation in isolate aggressiveness both within a single region (e.g., Brazil) (Zancan et al. 2015) and across a more widespread geographic region (e.g., Illinois and Argentina) (Kull et al. 2004). However, differences in bean isolate aggressiveness have been attributed to their location of origin (Otto-Hanson et al. 2011). Significant differences in aggressiveness on detached celery petioles were also reported in isolates collected from soybean, lettuce, and sunflower; sunflower isolates were determined to be the most aggressive (Durman et al. 2003). No differences due to isolate host or location of collection were detected in our study; however, obtaining a larger sample of isolates from each location would be necessary to clearly elucidate the effects of source and origin on isolate aggressiveness.

Our results also suggest that the location of collection did somewhat explain isolate OA production. However, other studies reported that sunflower isolates collected from China, Canada, and England varied significantly in OA production regardless of geographic origin (Li et al. 2008). In our collection, isolates from Poland $(n=10)$ and Michigan $(n=3)$ had the highest OA production and isolates collected in Minnesota $(n=3)$ had the lowest OA production; samples numbers, however, were low and may not be representative of the regional population. S. sclerotiorum isolates from lettuce, soybean, and sunflower also exhibited differences in OA production that were explained by the plant host origin (Durman et al. 2005). Durman et al. (2005) identified soybean isolates $(n=53)$ to exhibit the highest level of OA production, followed by sunflower $(n=19)$ and lettuce $(n=49)$. The host from which our isolates were collected also had a significant effect on in vitro OA production. Canola $(n=2)$ and dwarf everlast $(n=3)$ isolates consistently exhibited a higher mean OA

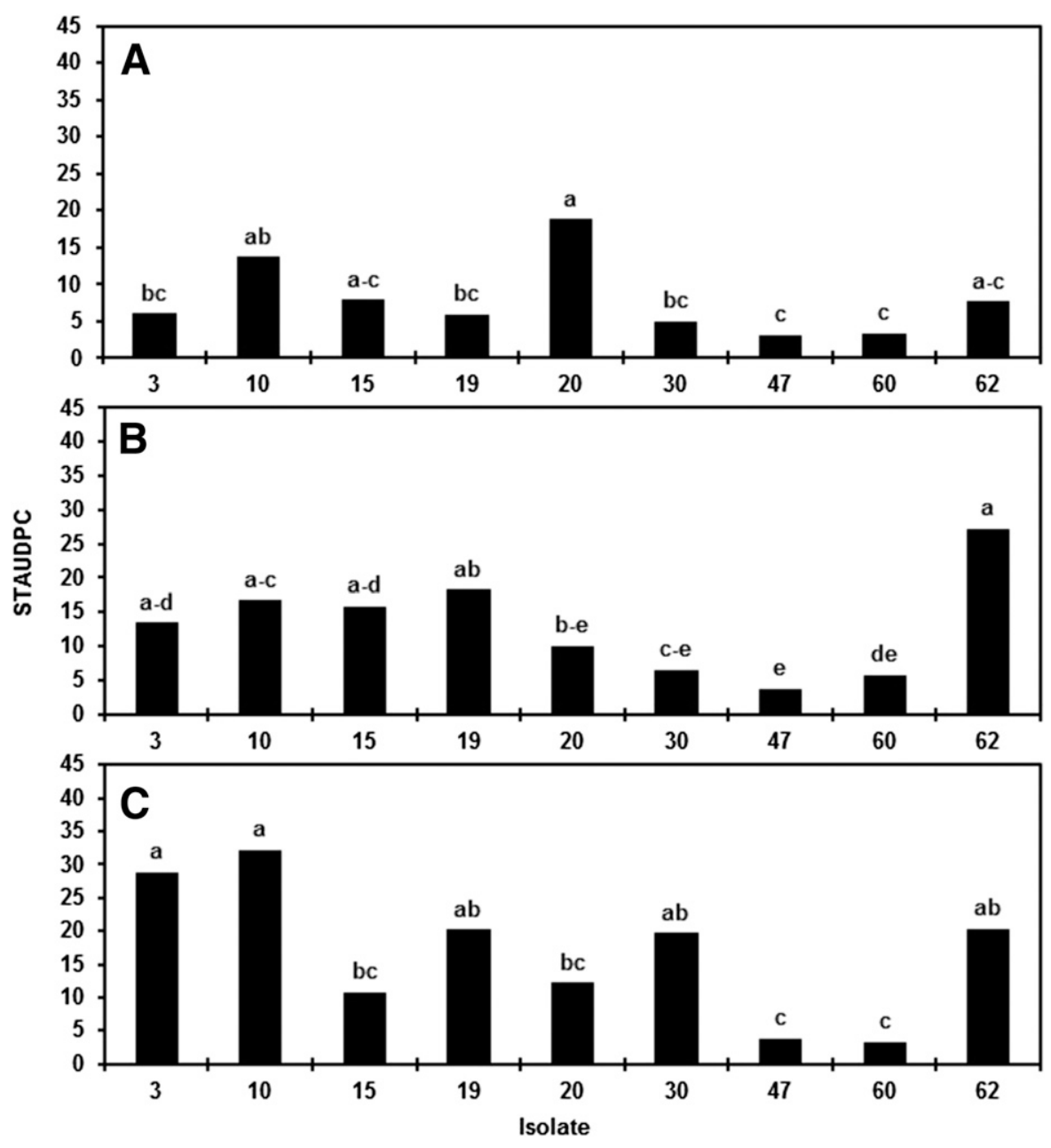

Fig. 7. Soybean germplasm reactions, measured as standardized areas under the disease progress curves (STAUDPC), to multiple Sclerotinia sclerotiorum isolates. Multiple isolates were used to challenge A, a strongly resistant germplasm line, 91-145; B, a moderately resistant line, 91-38; and C, a susceptible line, 91-44. Greenhouse evaluations were conducted by measuring stem lesions (in millimeters) every 1 to 3 days for 13 days postinoculation in runs 1 and 2 , or every 5 days for 15 days in run 3 . Runs 1, 2, and 3 are pooled and mean comparisons by recombinant inbred line were evaluated using Fisher's least significant difference. Bars designated by the same letter are not significantly different at the $\alpha=0.05$ significance level. The overall isolate-line interaction was significant $(P=0.04)$. Reactions with STAUDPC $\leq 10$ were considered resistant, STAUDPC $>10$ but $\leq 15$ were considered moderately susceptible, and STAUDPC $>15$ were considered susceptible. 
concentration than soybean $(n=29)$ isolates. As mentioned previously, the unbalanced nature of our $S$. sclerotiorum isolate collection most likely contributed to the inconsistencies between our findings and those of other studies. Although our results identified the diversity of OA production in S. sclerotiorum within several hosts and locations, the effect of host and location on OA production should be further investigated using a larger and more evenly distributed sample of isolates.

The differences in greenhouse and growth-room performance of isolates on Williams 82 soybean could be explained by simple light or temperature differences between the two environments. S. sclerotiorum infection in lettuce has been shown to be sensitive to temperature (Young et al. 2004). The growth room used in in initial aggressiveness studies was set to $22^{\circ} \mathrm{C}$ during the day and $18^{\circ} \mathrm{C}$ at night, with a 14-h photoperiod. The greenhouse temperature was approximately $24^{\circ} \mathrm{C}$ during the day; however, this environment may be more easily influenced by ambient temperatures. In addition, variability in photosynthetically active radiation levels have been shown to affect SSR development in both growth-room and field environments (Peltier and Grau 2008). Although light levels were not monitored in these experiments, it is evident that probable differences could explain the poor correlation between isolate performance in the growth room and greenhouse. However, as mentioned previously, the majority of the nine isolates used in the greenhouse experiments successfully clustered according to the aggressiveness groupings determined in previous growth-room evaluations. This suggests that any isolate-line interactions observed were not simply due to environmental differences.

The reactions of three soybean RIL to a diverse subset of nine $S$. sclerotiorum isolates were monitored in the greenhouse. The three RIL used in the greenhouse evaluations exhibited phenotypes consistent with the original selection designations (HR, MR, and S) during the development of these lines. Line 91-145 (HR) exhibited a highly resistant phenotype (STAUDPC $<10$ ) in our experiments. Although lines 91-38 (MR) and 91-44 (S) did not exhibit significantly different responses, line 91-44 displayed the highest STAUDPC mean ( $>15)$, which corresponds to the lowest level of resistance in our study. Ongoing studies of these germplasm lines recently suggested that the germplasm population from which our three lines are derived were typically more resistant than other populations despite having varying resistance levels (data not shown). Overall, the known and consistent levels of resistance of these lines enabled us to focus on phenotypic differences solely based on S. sclerotiorum isolate characteristics.

Using a representative subset of isolates, including the original isolate used for germplasm selection (number 30) and four strongly (numbers 10, 19, 20, and 62) and four mildly (numbers 3, 15, 47, and 60) aggressive isolates, differential resistance profiles were observed in the three soybean germplasm lines tested. Our results indicate that a comprehensive evaluation of these lines required the use of several $S$. sclerotiorum isolates. Resistance assessment using only the original isolate 30 , for example, would have determined line 91-44 (S) to be susceptible (STAUDPC > 15) and lines 91-38 (MR) and 91-145 (HR) to be resistant (STAUPDC < 10). However, using another isolate (e.g., number 20), we would consider 91-145 to be $\mathrm{S}$ and $91-44$ to be only MS $(10<$ STAUDPC $\leq 15)$. Using yet another aggressive isolate (e.g., number 62), we would consider 91-44 to be susceptible, 91-145 to be resistant, but 91-38 to be highly susceptible (STAUDPC $>20$ ). The use of a single mildly aggressive isolate (e.g., number 47) would suggest that all three germplasm lines were resistant. Furthermore, isolate number 15 is consistently one of the weakest isolates according to evaluations on Williams 82 (S). This isolate performed poorly on both our 91-44 (S) and 91-145 (HR) lines; however, it was among the most aggressive isolates on our line 91-38 (MR). Clearly, the resistant or susceptible outcome of these lines is directly dependent on the isolate used for evaluation. These results suggest that, regardless of the perceived aggressiveness of isolates or resistance level of the host, some isolates may be particularly equipped to overcome defense responses on a particular genotype, thus making screening for resistance using a single isolate challenging.
Our findings provide evidence that supports the use of a representative, well-characterized panel of $S$. sclerotiorum isolates, which includes both putatively strong and mildly aggressive isolates, especially when evaluating RIL. The differential profiles of these lines against various isolates suggest that either the lines confer different resistance mechanisms or different isolates potentially utilize different mechanisms to subvert host resistance. Soybean resistance mechanisms to $S$. sclerotiorum are not fully understood and quantitative trait loci mapping studies have shown that resistance to $S$. sclerotiorum is controlled by multiple genes throughout the soybean genome, revealing a complex genetic architecture of resistance to this pathogen (Huynh et al. 2010; Kim and Diers 2000). Thus, it is conceivable that the aggressiveness of a particular isolate may be dependent on the genetic make-up of the soybean host and, therefore, representative isolates should be used to encourage the selection of broadly resistant lines exhibiting multiple potential mechanisms. Use of such a tool may prevent selection of lines that express a single resistance mechanism that might be easily circumvented by a diverse population of S. sclerotiorum isolates, as might happen if just one perceived aggressive isolate is used.

The significant interaction between isolates and soybean lines observed in our experiment corroborates reported interactions between $S$. sclerotiorum isolates and genotypes of European red clover (Vleugels et al. 2013) and Brassica spp. (Garg et al. 2009). Our findings are in disagreement with other studies reporting insignificant interaction between cultivated host genotypes and S. sclerotiorum isolates in soybean (Auclair et al. 2004; Kull et al. 2004), alfalfa (Pratt and Rowe 1995), and Brassica spp. (Taylor et al. 2015). We hypothesize that the publicly available cultivars used in these studies (i) exhibited heterogeneous SSR reactions resulting in the failure to detect isolate-cultivar interactions and (ii) were only able to confer, at most, moderate resistance to SSR. We believe that we were able to detect this interaction as a result of the consistent responses in our highly inbred germplasm lines and our ability to incorporate a line exhibiting strong SSR resistance.

Overall, the high level of diversity in S. sclerotiorum isolate aggressiveness within our collection was unexplained by host or location of collection. Isolate aggressiveness was also not fully explained by either in vitro radial growth or in vitro OA production. As a result, a representative subset of isolates exhibiting varying levels of aggressiveness was selected to investigate soybean germplasm reactions to multiple isolates, and differential host profiles were observed. Our findings clearly demonstrate the necessity of using multiple isolates to determine the resistance profile of soybean genotypes. These isolates should be selected based on results from thorough diversity studies which consider essential components of S. sclerotiorum isolate aggressiveness. These results strongly suggest using a representative panel of well-characterized S. sclerotiorum isolates in comprehensive SSR resistance evaluations in soybean.

\section{Acknowledgments}

We thank C. Bradley, M. Chilvers, A. Baturo-Ciesniewska, C. Fritz, K. Lackermann, and D. Malvick for the field S. sclerotiorum isolate collections. This research was graciously funded by the Wisconsin Soybean Marketing Board and through the Science and Medicine Graduate Research Scholars program at the University of Wisconsin-Madison.

\section{Literature Cited}

Aldrich-Wolfe, L., Travers, S., and Nelson, B. D. 2015. Genetic variation of Sclerotinia sclerotiorum from multiple crops in the north central United States. PLoS One 10:e139188.

Amselem, J., Cuomo, C. A., van Kan, J. A. L., Viaud, M., Benito, E. P., Couloux, A., Coutinho, P. M., de Vries, R. P., Dyer, P. S., Fillinger, S., Fournier, E., Gout, L., Hahn, M., Kohn, L., Lapalu, N., Plummer, K. M., Pradier, J.-M., Quévillon, E., Sharon, A., Simon, A., ten Have, T., Tudzynski, B., Tudzynski, P., Wincker, P., Andrew, M., Anthouard, V., Beever, R. E., Beffa, R., Benoit, I., Bouzid, O., Brault, B., Chen, Z., Choquer, M., Collémare, J., Cotton, P., G. Danchin, E. G., Da Silva, C., Gautier, A., Giraud, C,. Giraud, T., Gonzalez, C., Grossetete, S. Güldener, U., Henrissat, B., Howlett, B. J., Kodira, C., Kretschmer, M., Lappartient, A., Leroch, M., Levis, C., Mauceli, E., Neuvéglise, C., Oeser, B., Pearson, M., Poulain, J., Poussereau, N., Quesneville, H., Rascle, C., Schumacher, J., Ségurens, B., Sexton, A., Silva, E., Sirven, C., Soanes, D. M. Talbot, N. J., Templeton, M., Yandava, C., Yarden, O., Zeng, Q., Rollins, J. A., 
Lebrun, M.-H., and Dickman, M. 2011. Genomic analysis of the necrotrophic fungal pathogens Sclerotinia sclerotiorum and Botrytis cinerea. PLoS Genet. 7:e1002230.

Anderson, J. B., and Kohn, L. M. 1995. Clonality in soilborne, plant-pathogenic fungi. Annu. Rev. Phytopathol. 33:369-391.

Auclair, J., Boland, G. J., Kohn, L. M., and Rajcan, I. 2004. Genetic interactions between Glycine max and Sclerotinia sclerotiorum using a straw inoculation method. Plant Dis. 88:891-895.

Bateman, D. F., and Beer, S. V. 1965. Simultaneous production and synergistic action of oxalic acid and polygalacturonase during pathogenesis by Sclerotium rolfsii. Phytopathology 55:204-211.

Baturo-Ciesniewska, A., Groves, C. L., Albrecht, K. A., Grau, C. R., Willis, D. K., and Smith, D. L. Molecular identification of Sclerotinia trifoliorum and Sclerotinia sclerotiorum isolates from the United States and Poland. Plant Dis. In press. doi:10.1094/PDIS-06-16-0896-RE

Ben-Yephet, Y., and Bitton, S. 1985. Use of a selective medium to study the dispersal of ascospores of Sclerotinia sclerotiorum. Phytoparasitica 13:33-40.

Boland, G. J., and Hall, R. 1986. Growthroom evaluation of soybean cultivars for resistance to Sclerotinia sclerotiorum. Can. J. Plant Sci. 66:559-564.

Boland, G. J., and Hall, R. 1987. Evaluating soybean cultivars for resistance to Sclerotinia sclerotiorum under field conditions. Plant Dis. 71:934-936.

Boland, G. J., and Hall, R. 1994. Index of plant hosts of Sclerotinia sclerotiorum. Can. J. Plant Pathol. 16:93-108.

Campbell, C. L., and Madden, L. V. 1990. Introduction to Plant Disease Epidemiology. John Wiley and Sons, New York.

Cessna, S. G., Sears, V. E., Dickman, M. B., and Low, P. S. 2000. Oxalic acid, a pathogenicity factor for Sclerotinia sclerotiorum, suppresses the oxidative burst of the host plant. Plant Cell 12:2191-2199.

Durman, S. B., Menéndez, A. B., and Godeas, A. M. 2003. Mycelial compatibility groups in Buenos Aires field populations of Sclerotinia sclerotiorum (Sclerotiniaceae). Aust. J. Bot. 51:421-427.

Durman, S. B., Menendez, A. B., and Godeas, A. M. 2005. Variation in oxalic acid production and mycelial compatibility within field populations of Sclerotinia sclerotiorum. Soil Biol. Biochem. 37:2180-2184.

Garg, H., Kohn, L. M., Andrew, M., Li, H., Sivasithamparam, K., and Barbetti, M. J. 2009. Pathogenicity of morphologically different isolates of Sclerotinia sclerotiorum with Brassica napus and B. juncea genotypes. Eur. J. Plant Pathol. 126:305-315.

Godoy, G., Steadman, J., Dickman, M., and Dam, R. 1990. Use of mutants to demonstrate the role of oxalic acid in pathogenicity of Sclerotinia sclerotiorum on Phaseolus vulgaris. Physiol. Mol. Plant Pathol. 37:179-191.

Grau, C., Radke, V., and Gillespie, F. 1982. Resistance of soybean cultivars to Sclerotinia sclerotiorum. Plant Dis. 66:506-508.

Guimarães, R. L., and Stotz, H. U. 2004. Oxalate production by Sclerotinia sclerotiorum deregulates guard cells during infection. Plant Physiol. 136:3703-3711.

Guyon, K., Balagué, C., Roby, D., and Raffaele, S. 2014. Secretome analysis reveals effector candidates associated with broad host range necrotrophy in the fungal plant pathogen Sclerotinia sclerotiorum. BMC Genomics 15:336.

Hartman, G. L., Kull, L., and Huang, Y. H. 1998. Occurrence of Sclerotinia sclerotiorum in soybean fields in East-Central Illinois and enumeration of inocula in soybean seed lots. Plant Dis. 82:560-564.

Hoffman, D. D., Diers, B. W., Hartman, G. L., Nickell, C. D., Nelson, R. L., Pedersen, W. L., Cober, E. R., Graef, G. L., Steadman, J. R., Grau, C. R., Nelson, B. D., del Rio, L. E., Helms, T., Anderson, T., Poysa, V., Rajcan, I., and Stienstra, W. C. 2002. Selected soybean plant introductions with partial resistance to Sclerotinia sclerotiorum. Plant Dis. 86:971-980.

Huynh, T. T., Bastien, M., Iquira, E., Turcotte, P., and Belzile, F. 2010. Identification of QTLs associated with partial resistance to white mold in soybean using field-based inoculation. Crop Sci. 50:969-979.

Kabbage, M., Williams, B., and Dickman, M. B. 2013. Cell death control: The interplay of apoptosis and autophagy in the pathogenicity of Sclerotinia sclerotiorum. PLoS Pathog. 9:e1003287.

Kabbage, M., Yarden, O., and Dickman, M. B. 2015. Pathogenic attributes of Sclerotinia sclerotiorum: Switching from a biotrophic to necrotrophic lifestyle. Plant Sci. 233:53-60.

Kim, H. S., and Diers, B. W. 2000. Inheritance of partial resistance to Sclerotinia stem rot in soybean. Crop Sci. 40:55-61.

Kim, H. S., Hartman, G. L., Manandhar, J. B., Graef, G. L., Steadman, J. R., and Diers, B. W. 2000. Reaction of soybean cultivars to Sclerotinia stem rot in field, greenhouse, and laboratory evaluations. Crop Sci. 40:665-669.

Koenning, S. R., and Wrather, J. A. 2010. Suppression of soybean yield potential in the continental United States by plant diseases from 2006 to 2009. Online publication. Plant Health Prog. doi:10.1094/PHP-2010-1122-01-RS

Koga, L. J., Bowen, C. R., Godoy, C. V., De Oliveira, M. C. N., and Hartman, G. L. 2014. Mycelial compatibility and aggressiveness of Sclerotinia sclerotiorum isolates from Brazil and the United States. Pesqui. Agropecu. Bras. 49:265-272.
Kohn, L. M., Carbone, I., and Anderson, J. B. 1990. Mycelial interactions in Sclerotinia sclerotiorum. Exp. Mycol. 14:255-267.

Kohn, L. M., Stasovski, E., Carbone, I., Royer, J., and Anderson, J. B. 1991 Mycelial incompatibility and molecular markers identify genetic variability in field populations of Sclerotinia sclerotiorum. Phytopathology 81:480485.

Kull, L. S., Pedersen, W. L., Palmquist, D., and Hartman, G. L. 2004. Mycelial compatibility grouping and aggressiveness of Sclerotinia sclerotiorum. Plant Dis. 88:325-332.

Lehner, M. S., Lima, R. C., Carneiro, J. E. S., Paula, T. J., Junior., Vieira, R. F., and Mizubuti, E. S. G. 2016. Similar aggressiveness of phenotypically and genotypically distinct isolates of Sclerotinia sclerotiorum. Plant Dis. 100: 360-366.

Li, Z., Zhang, Y., Li, R., and Fernando, W. G. D. 2008. Mycelial compatibility group and pathogenicity variation of Sclerotinia sclerotiorum populations in sunflower from China, Canada and England. Plant Pathol. 7:131-139.

Liang, X., Liberti, D., Li, M., Kim, Y.-T., Hutchens, A., Wilson, R., and Rollins, J. A. 2015. Oxaloacetate acetylhydrolase gene mutants of Sclerotinia sclerotiorum do not accumulate oxalic acid, but do produce limited lesions on host plants. Mol. Plant Pathol. 16:559-571.

Maxwell, D. P., and Lumsden, R. D. 1970. Oxalic acid production by Sclerotinia sclerotiorum in infected bean and in culture. Phytopathology 60:13951398.

Nickell, C. D., Noel, G. R., Bernard, R. L., Thomas, D. J., and Frey, K. 1994 Registration of soybean germplasm line LN89-5717, resistant to soybean cyst nematode. Crop Sci. 34:1133-1134.

Noyes, R. D., and Hancock, J. G. 1981. Role of oxalic acid in the Sclerotinia wilt of sunflower. Physiol. Plant Pathol. 18:123-132.

Otto-Hanson, L., Steadman, J. R., Higgins, R., and Eskridge, K. M. 2011 Variation in Sclerotinia sclerotiorum bean isolates from multisite resistance screening locations. Plant Dis. 95:1370-1377.

Peltier, A. J., Bradley, C. A., Chilvers, M. I., Malvick, D. K., Mueller, D. S., Wise, K. A., and Esker, P. D. 2012. Biology, yield loss and control of Sclerotinia stem rot of soybean. J. Integr. Pest Manage. 3:1-7.

Peltier, A. J., and Grau, C. R. 2008. The influence of light on relationships between Sclerotinia stem rot of soybean in field and controlled environments. Plant Dis. 92:1510-1514

Petrofeza, S., and Nasser, L. C. B. 2012. Case study: Sclerotinia sclerotiorum: Genetic diversity and disease control. In: The Molecular Basis of Plant Genetic Diversity. M. Caliskan, ed. Online publication. InTech.

Pottinger, B. M., Ridgway, H. J., Bourd̂t, G. W., and Stewart, A. 2008 Influence of storage method on the viability, mycelial growth and pathogenicity of Sclerotinia sclerotiorum sclerotia. Australas. Plant Pathol. 37:609-614.

Pratt, R. G., and Rowe, D. E. 1995. Comparative pathogenicity of isolates of Sclerotinia trifoliorum and S. sclerotiorum on alfalfa cultivars. Plant Dis. 79: 474-477.

Taylor, A., Coventry, E., Jones, J. E., and Clarkson, J. P. 2015. Resistance to a highly aggressive isolate of Sclerotinia sclerotiorum in a Brassica napus diversity set. Plant Pathol. 64:932-940.

USDA-NASS. 2011. United States soybean prices. United States Department of Agriculture-National Agricultural Statistics Service (USDA-NASS), Washington, DC.

Vleugels, T., Baert, J., and van Bockstaele, E. 2013. Morphological and pathogenic characterization of genetically diverse Sclerotinia isolates from European red clover crops (Trifolium pratense L.). J. Phytopathol. 161: 254-262.

Wegulo, S. N., Yang, X. B., and Martinson, C. A. 1998. Soybean cultivar responses to Sclerotinia sclerotiorum in field and controlled environment studies. Plant Dis. 82:1264-1270.

Willetts, H. J., and Wong, J. A.-L. 1980. The biology of Sclerotinia sclerotiorum, S. trifoliorum, and S. minor with emphasis on specific nomenclature. Bot. Rev. 46:101-165.

Williams, B., Kabbage, M., Kim, H.-J., Britt, R., and Dickman, M. B. 2011 Tipping the balance: Sclerotinia sclerotiorum secreted oxalic acid suppresses host defenses by manipulating the host redox environment. PLoS Pathog. 7:e1002107.

Wrather, A., and Koenning, S. 2009. Effects of diseases on soybean yields in the United States 1996 to 2007. Online publication. Plant Health Prog. doi:10.1094/ PHP-2009-0401-01-RS

Young, C. S., Clarkson, J. P., Smith, J. a., Watling, M., Phelps, K., and Whipps, J. M. 2004. Environmental conditions influencing Sclerotinia sclerotiorum infection and disease development in lettuce. Plant Pathol. 53:387-397.

Zancan, W. L. A., Steadman, J. R., Higgins, R., Jhala, R., and Machado, J. da C. 2015. Genetic and aggressiveness variation among Sclerotinia sclerotiorum dry bean isolates from Brazil fields. Biosci. J. 31:1143-1151. 